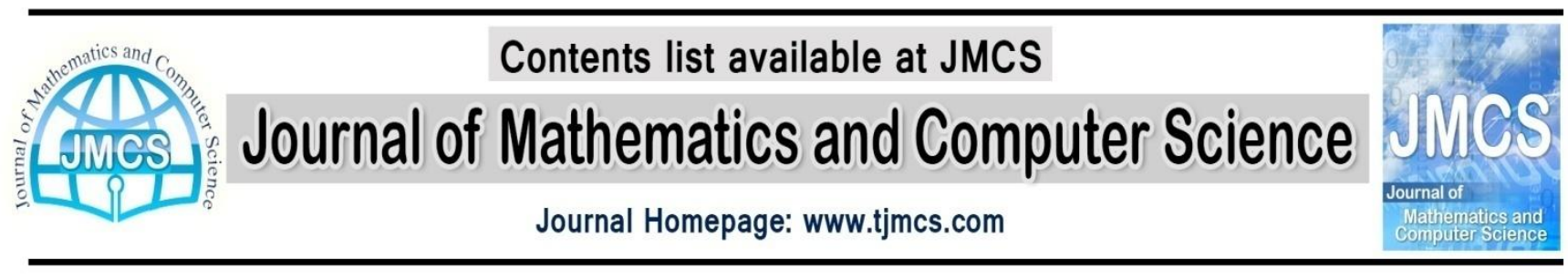

\title{
Numerical solution for nonlinear-quadratic switching control systems with time delay
}

\author{
Fateme Ghomanjani, \\ Mohammad Hadi Farahi, \\ Ali Vahidian Kamyad \\ Department of Mathematics, Ferdowsi University of Mashhad, Mashhad, Iran
}

E-mail:fatemeghomanjani@gmail.com

Article history:

Received August 2013

Accepted November 2013

Available online November 2013

\begin{abstract}
This paper contributes an efficient numerical approach for optimal control of switched system with time delay via Bezier curves. A simple transformation is first used to map the optimal control problem with varying switching times into a new optimal control problem with fixed switching times. Then, the Bezier curves is used to approximate the optimal control problem a NLP. The NLP could be solved by using known algorithms.
\end{abstract}

Keywords: switched systems; Bezier control points; time delay systems; dynamical system.

\section{Introduction}

A switched system can be explained by numerous modes or state variable descriptions and a switching status that triggers transitions between the modes. Typically, the switching status relies on state or time events and aims to improve the performance of a system. The Switched system has detected many applications, for instance, in air traffic management, manufacturing system, embedded auto controllers and vibration controls (see [2], [5], [8], [10], [16], and [20]).

Switched systems provide a suitable mathematical model for such processes, and their stability analysis is important for both theoretical and practical reasons. Margaliot [21] reviewed a specific approach for stability analysis based on using variational principles to characterize the "most unstable" solution of the switched system. Margaliot [21] also discussed a link between the variational approach and the stability analysis of switched systems using Lie-algebraic considerations. Both approaches required the use of sophisticated tools from many different fields of applied mathematics.

For switched systems with linear component systems, the defect of optimal control switching has absorbed several researchers attention recently. For instance, Egerstedt, et al. [5] presented such an accurate method and algorithm which is able to compute the minimum number of switching for changing from one state to another and $\mathrm{Xu}$, et al. [29] addressed the problem of determining the switched instants. 
Some of the recent sheets on the accurate switching problems make state feedback solution. Assuming a known and finite switching sequence, a state feedback controller was designed by Giua, et al. [13]. The controller based on a numerically constructed switching table that indicates points in the state space where switching should take place. The optimization problem was later generalized by Bemporad, et al. [2] by taking both the switching instants and the switching sequence as decision variables.

$\mathrm{Xu}$, et al. [28] continued their recent study on practical stabilizability of discrete-time (DT) switched

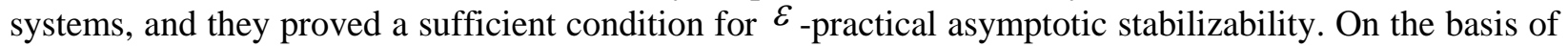
their approach, they also presented several new sufficient conditions for global $\varepsilon_{\text {-practical asymptotic }}$ stabilizability of such a class of systems.

Piecewise polynomial functions are often used to represent the approximated solution in the numerical solution of differential equations, (see [1], [3], [4], [6], [7], [15], [17], and [26]). B-splines, due to numerical stability and arbitrary order of accuracy, have become popular tools for solving differential equations (where Bezier form is a special case of B-splines). There are many papers and books deal with the Bezier curves or surface techniques. Harada, and Nakamae [14], Nürnberger, and Zeilfelder [23] used the Bezier control points in approximated data and functions. Zheng, et al. [30] proposed the use of control points of the Bernstein-Bezier form for solving differential equations numerically and also Evrenosoglu, and Somali [6] were used this approach for solving singular perturbed two points boundary value problems. The Bezier curves are used in solving partial differential equations, as well, Wave and Heat equations are solvable in Bezier form, (see [1], and [19]). Bezier curves are used for solving dynamical systems, (see [9]), also the Bezier control points method is used for solving delay differential equation (see [11]). Some other applications of the Bezier functions and control points are found in [4], and [25], that are used in computer aided geometric design and image compression. The use of the Bezier curves for solving nonlinear-quadratic switching control with time delay is a novel idea. Although the method is very easy to use and straightforward, the obtained results are satisfactory (see the numerical result).

We suggest a similar technique which is used in [11], [12] and [30] for solving nonlinear-quadratic switching control with time delay.

The remainder of this paper is organized as follows: in Section 2, statement of the problem will be introduced. Problem approximation will be described in Section 3. In Section 4, we provide one numerical example illustrating the accuracy of the presented method. Finally, Section 5 will give a brief conclusion.

\section{Statement of the problem}

A switched system composing of following subsystems is considered as:

$$
\frac{d \mathbf{x}(t)}{d t}=F_{i}(t, \mathbf{x}(t), \mathbf{u}(t)), i \in I=\{1,2, \ldots, N\},
$$

where $N$ is a positive integer, and $\mathbf{x}(t) \in \mathrm{R}^{p}, \mathbf{u}(t) \in \mathrm{R}^{m}$ and for each $i \in I, F_{i}: \mathrm{R}^{p+m+1} \rightarrow \mathrm{R}^{p}$ is continuously differentiable with respect to its arguments. A switching sequence with $t \in\left[t_{0}, t_{f}\right]$ adjusts the order of active subsystems, and it is defined as:

$$
\left.\sigma=\left\{\left(T_{0}, i_{0}\right),\left(T_{1}, i_{1}\right)\right), \ldots,\left(T_{K}, i_{K}\right)\right\}
$$

where $0 \leq K<\infty, t_{0}=T_{0} \leq T_{1} \leq \ldots \leq T_{K} \leq t_{f}$, and $i_{k} \in I$, for $k=0,1, \ldots, K$. Here, $\left(T_{k}, i_{k}\right)$ indicates that at instant $T_{k}$, the system switches from subsystem $i_{k-1}$ to subsystem $i_{k}$; therefore subsystem $i_{k}$ is active during time interval $\left[T_{k}, T_{k+1}\right)\left(\left[T_{K}, T_{f}\right]\right.$ if $\left.k=K\right)$. In order a switched system to be well-behaved, only non zeno sequences are considered which switch at most a finite number of times in $\left[t_{0}, t_{f}\right]$, though different sequences may be different numbers of switchings. If $\sigma$ is regarded as a 
discrete input, then the overall control input on the system is the pair $(\sigma, u)$. It is maintained that the discriminated factor for a switched system from a general hybrid system is its continuous state which doesn't represent jumps at switching instants. Hence, the computation of continuous inputs becomes favorable via the application of conventional optimal control methods.

Now, without loss of generality, the paper is considered the following form

$$
\begin{aligned}
& \min I=\varphi\left(\mathbf{x}\left(t_{f}\right)\right)+\int_{t_{0}}^{t} f(\mathbf{x}(t), \mathbf{u}(t)) d t, \\
& \text { s.t. } \\
& \frac{d \mathbf{x}(t)}{d t}=F\left(\mathbf{x}(t), \mathbf{u}(t), x_{1}\left(t-\tau_{1}\right), \ldots, x_{p}\left(t-\tau_{p}\right), u_{1}\left(t-\eta_{1}\right), \ldots, u_{m}\left(t-\eta_{m}\right)\right), \\
& \quad t \in\left[t_{0}, T_{1}\right), \quad(a) \\
& \frac{d \mathbf{x}(t)}{d t}=G\left(\mathbf{x}(t), \mathbf{u}(t), x_{1}\left(t-\tau_{1}\right), \ldots, x_{p}\left(t-\tau_{p}\right), u_{1}\left(t-\eta_{1}\right), \ldots, u_{m}\left(t-\eta_{m}\right)\right), \\
& \frac{d \mathbf{x}(t)}{d t}=\left[T_{1}, T_{2}\right), \quad(b) \\
& t \in\left[\left(\mathbf{x}(t), \mathbf{u}(t), x_{1}\left(t-\tau_{1}\right), \ldots, x_{p}\left(t-\tau_{p}\right), u_{1}\left(t-\eta_{1}\right), \ldots, u_{m}\left(t-\eta_{m}\right)\right),\right. \\
& \mathbf{x}(t)=\phi(t), \quad t \leq t_{0}, \\
& \mathbf{u}(t)=\psi(t), \quad t \leq t_{0},
\end{aligned}
$$

where $\quad \mathbf{x}(t)=\left(x_{1}(t) \ldots x_{p}(t)\right)^{T} \in \mathrm{R}^{p}, \quad \mathbf{u}(t)=\left(u_{1}(t) \ldots u_{m}(t)\right)^{T} \in \mathrm{R}^{m}, \phi(t)=\left(\phi_{1}(t) \ldots \phi_{p}(t)\right)^{T}, \quad$ and $\boldsymbol{\psi}(t)=\left(\psi_{1}(t) \ldots \psi_{m}(t)\right)^{T}$ are known vector functions, and $t_{0}$ and $t_{f}$ are known values in $\mathrm{R}$, and $\tau_{i}$ 's and $\eta_{j}$ 's, $(i=1,2, \ldots, p, j=1,2, \ldots, m)$ are non-negative constant time delays.

We assume $F()=.\left(f_{1}(.) \ldots f_{p}(.)\right)^{T}, G()=.\left(g_{1}(.) \ldots g_{p}(.)\right)^{T}$ and $Q()=.\left(q_{1}(.) \ldots q_{p}(.)\right)^{T}$ are vector functions, which their elements respectively assume to be polynomials defined on $\left[t_{0}, T_{1}\right),\left[T_{1}, T_{2}\right)$ and $\left[T_{2}, t_{f}\right]$. We need to impose the continuity condition on $\mathbf{x}(t)$ and its first derivative which these constraints are appeared in Section 3.

Without loss of generality just for simplicity, the case of three subsystems is considered in which the subsystems $(a),(b)$ and $(c)$ in (3) are active in $t \in\left[t_{0}, T_{1}\right), t \in\left[T_{1}, T_{2}\right)$ and $t \in\left[T_{2}, t_{f}\right]$, respectively ( $T_{1}$ and $T_{2}$ are switching instants which have to be determined) (see [29]).

Problem (3) can be converted into the following problem. Variables $y_{1}$ and $y_{2}$ are introduced corresponding to switching instants $T_{1}$ and $T_{2}$. Let $y_{1}$ and $y_{2}$ satisfy

$$
\begin{aligned}
& \frac{d y_{1}}{d t}=0, \\
& y_{1}(0)=T_{1}, \\
& \frac{d y_{2}}{d t}=0, \\
& y_{2}(0)=T_{2} .
\end{aligned}
$$

A new independent time variable $\zeta$ is introduced. Hence, a piecewise linear relationship between $t$ and $\zeta$ is: 


$$
t=\left\{\begin{array}{cc}
t_{0}+\left(y_{1}-t_{0}\right) \zeta, & 0 \leq \zeta<1 \\
y_{1}+\left(y_{2}-y_{1}\right)(\zeta-1), & 1 \leq \zeta<2 \\
y_{2}+\left(t_{f}-y_{2}\right)(\zeta-2), & 2 \leq \zeta \leq 3 .
\end{array}\right.
$$

Obviously, $\zeta=0$ corresponds $t=t_{0}, \zeta=1$ to $t=T_{1}, \zeta=2$ to $t=T_{2}$, and $\zeta=3$ to $t=t_{f}$. By inputting $y_{1}, y_{2}, \zeta, F(),. G($.$) and Q($.$) in (a),(b)$ and $(c)$ in (3) are converted into $F_{1}(),. G_{1}($. and $Q_{1}$ (.) respectively, and the problem (3) is converted into following equivalent equations:

$$
\begin{aligned}
& \frac{d \mathbf{x}(\zeta)}{d \zeta}=\left(y_{1}-t_{0}\right) F_{1}\left(\mathbf{x}(\zeta), \mathbf{u}(\zeta), x_{1}\left(\zeta-\tau_{1}\right), \ldots, x_{p}\left(\zeta-\tau_{p}\right), u_{1}\left(\zeta-\eta_{1}\right), \ldots, u\left(\zeta-\eta_{m}\right)\right), \\
& \frac{d y_{1}}{d \zeta}=0
\end{aligned}
$$

in the interval $\zeta \in[0,1)$, and

$$
\begin{aligned}
\frac{d \mathbf{x}(\zeta)}{d \zeta}=\left(y_{2}-y_{1}\right) G_{1} & \left(\mathbf{x}(\zeta), \mathbf{u}(\zeta), x_{1}\left(\zeta-\tau_{1}\right), \ldots, x_{p}\left(\zeta-\tau_{p}\right), u_{1}\left(\zeta-\eta_{1}\right), \ldots, u\left(\zeta-\eta_{m}\right)\right) \\
\frac{d y_{1}}{d \zeta} & =0 \\
\frac{d y_{2}}{d \zeta} & =0
\end{aligned}
$$

in the interval $\zeta \in[1,2)$, and

$$
\begin{gathered}
\frac{d \mathbf{x}(\zeta)}{d \zeta}=\left(t_{f}-y_{2}\right) Q_{1}\left(\mathbf{x}(\zeta), \mathbf{u}(\zeta), x_{1}\left(\zeta-\tau_{1}\right), \ldots, x_{p}\left(\zeta-\tau_{p}\right), u_{1}\left(\zeta-\eta_{1}\right), \ldots, u\left(\zeta-\eta_{m}\right)\right) \\
\frac{d y_{2}}{d \zeta}=0
\end{gathered}
$$

in the interval $\zeta \in[2,3]$, and

$$
\begin{aligned}
& \mathbf{x}_{j}\left(\zeta+t_{0}\right)=\phi\left(\zeta+t_{0}\right), \quad \zeta \leq 0, \\
& \mathbf{u}_{j}\left(\zeta+t_{0}\right)=\boldsymbol{\psi}\left(\zeta+t_{0}\right), \quad \zeta \leq 0 .
\end{aligned}
$$

Now, the target is to finding $y_{1}, y_{2}$ and $\mathbf{u}(\zeta), \zeta \in[0,3]$ such that the cost function

$$
\begin{aligned}
& I=\varphi(\mathbf{x}(3))+\int_{0}^{1}\left(y_{1}-t_{0}\right) L(\mathbf{x}(\zeta), \mathbf{u}(\zeta)) d \zeta \\
& +\int_{1}^{2}\left(y_{2}-y_{1}\right) L(\mathbf{x}(\zeta), \mathbf{u}(\zeta)) d \zeta+\int_{2}^{3}\left(t_{f}-y_{2}\right) L(\mathbf{x}(\zeta), \mathbf{u}(\zeta)) d \zeta
\end{aligned}
$$

is to be minimized.

Remark 2.1The equivalent problem (9)-(17) provides us with no more varying switching instants, and consequently, it is conventional. Since $y_{1}$ and $y_{2}$ are actually unknown constants when $\zeta \in[0,3]$; 
hence, $y_{1}$ and $y_{2}$ are regarded as unknown parameters for optimal control problem with cost function (17) and subsystems (9)-(16).

\section{Problem approximation}

Consider the optimal control of time varying system (9)-(16) by the cost function (17), with delays in state and control. Divide the interval $[0,3]$ into a set of grid points such that

$$
\zeta_{i}=0+i h, i=0,1, \ldots, 3 k,
$$

where $h=\frac{3}{3 k}$, and $k$ is a positive integer. Let $S_{j}=\left[\zeta_{j-1}, \zeta_{j}\right]$ for $j=1,2, \ldots, 3 k$. Here, for $\zeta \in S_{j}$ the above optimal control problem can be decomposed to the following suboptimal control problem:

$$
\begin{aligned}
& \min I_{j}=C_{j}+I_{\text {first }}+I_{\text {second }}+I_{\text {third }} \\
& \text { s.t. } \\
& I_{\text {first }}=\int_{\zeta_{j-1}}^{\zeta_{j}}\left(y_{1}-t_{0}\right) L\left(\mathbf{x}_{j}(\zeta), \mathbf{u}_{j}(\zeta)\right) d \zeta, j=1,2, \ldots, k, \zeta \in[0,1), \\
& I_{\text {second }}=\int_{\zeta_{j-1}}^{\zeta_{j}}\left(y_{2}-y_{1}\right) L\left(\mathbf{x}_{j}(\zeta), \mathbf{u}_{j}(\zeta)\right) d \zeta, j=k+1, \ldots, 2 k, \zeta \in[1,2) \text {, } \\
& I_{\text {third }}=\int_{\zeta_{j-1}}^{\zeta_{j}}\left(t_{f}-y_{2}\right) L\left(\mathbf{x}_{j}(\zeta), \mathbf{u}_{j}(\zeta)\right) d \zeta, j=2 k+1, \ldots, 3 k, \zeta \in[2,3] \text {, } \\
& \frac{d \mathbf{x}_{j}}{d \zeta}=\chi_{[0,1)}\left(y_{1}-t_{0}\right) F_{1}\left(\mathbf{x}(\zeta), \mathbf{u}(\zeta), x_{1}^{-k_{1}^{1}+j}\left(\zeta-\tau_{1}\right), \ldots, x_{p}^{-k_{1}^{p}+j}\left(\zeta-\tau_{p}\right)\right. \\
& \left., u_{1}^{-k_{2}^{1}+j}\left(\zeta-\eta_{1}\right), \ldots, u_{m}^{-k_{2}^{m}+j}\left(\zeta-\eta_{m}\right)\right), j=1,2, \ldots, k, \zeta \in S_{j}, \\
& \frac{d \mathbf{x}_{j}}{d \zeta}=\chi_{[1,2)}\left(y_{2}-y_{1}\right) G_{1}\left(\mathbf{x}(\zeta), \mathbf{u}(\zeta), x_{1}^{-k_{1}^{1}+j}\left(\zeta-\tau_{1}\right), \ldots, x_{p}^{-k_{1}^{p}+j}\left(\zeta-\tau_{p}\right)\right. \\
& \left., u_{1}^{-k_{2}^{1}+j}\left(\zeta-\eta_{1}\right), \ldots, u_{m}^{-k_{2}^{m}+j}\left(\zeta-\eta_{m}\right)\right), j=k+1, \ldots, 2 k, \zeta \in S_{j}, \\
& \frac{d \mathbf{x}_{j}}{d \zeta}=\chi_{[2,3]}\left(t_{f}-y\right) Q_{1}\left(\mathbf{x}(\zeta), \mathbf{u}(\zeta), x_{1}^{-k_{1}^{1}+j}\left(\zeta-\tau_{1}\right), \ldots, x_{p}^{-k_{1}^{p}+j}\left(\zeta-\tau_{p}\right)\right. \\
& \left., u_{1}^{-k_{2}^{1}+j}\left(\zeta-\eta_{1}\right), \ldots, u_{m}^{-k_{2}^{m}+j}\left(\zeta-\eta_{m}\right)\right), j=2 k+1, \ldots, 3 k, \zeta \in S_{j}, \\
& \frac{d y_{1}}{d \zeta}=0, \zeta \in[0,2] \\
& \frac{d y_{2}}{d \zeta}=0, \zeta \in[1,3] \\
& \mathbf{x}_{j}\left(\zeta+t_{0}\right)=\varphi\left(\zeta+t_{0}\right), \zeta \leq 0 \\
& \mathbf{u}_{j}\left(\zeta+t_{0}\right)=\boldsymbol{\psi}\left(\zeta+t_{0}\right), \zeta \leq 0 \text {, }
\end{aligned}
$$


where $\mathbf{x}_{j}(\zeta)=\left(x_{1}^{j}(\zeta) \ldots x_{p}^{j}(\zeta)\right)^{T}$, and $\mathbf{u}_{j}(\zeta)=\left(u_{1}^{j}(\zeta) \ldots u_{m}^{j}(\zeta)\right)^{T}$ are respectively vectors of $\mathbf{x}(\zeta)$ and $\mathbf{u}(\zeta)$ which are considered in $\zeta \in S_{j}$, and $\chi_{[0,1)}, \chi_{[1,2)}$ and $\chi_{[2,3]}$ are respectively characteristic functions for $\zeta \in[0,1), \zeta \in[1,2)$ and $\zeta \in[2,3]$, we mention that $x_{i}^{-k_{1}^{i}+j}\left(\zeta-\tau_{i}\right)$; $1 \leq i \leq p$, is the $\mathrm{i}$-th component $\left(x_{1}^{-k_{1}^{1}+j}\left(\zeta-\tau_{1}\right) \ldots x_{p}^{-k_{1}^{p}+j}\left(\zeta-\tau_{p}\right)\right)^{T}$ where $\left(\zeta-\tau_{i}\right) \in\left[\zeta_{-k_{1}^{i}+j-1}, \zeta_{-k_{1}^{i}+j}\right]$, and $u_{i}^{-k_{2}^{i}+j}\left(\zeta-\eta_{i}\right) ; 1 \leq i \leq m$ has the same definition as well. Also

$$
\begin{aligned}
& C_{j}=\left\{\begin{array}{cc}
\varphi(\mathbf{x}(3)) & j=3 k \\
0 & j \neq 3 k
\end{array},\right. \\
& k_{1}^{i}=\left\{\begin{array}{cl}
\frac{\tau_{i}}{h} & \frac{\tau_{i}}{h} \in \mathrm{N} \\
\left(\left[\frac{\tau_{i}}{h}\right]+1\right) & \frac{\tau_{i}}{h} \notin \mathrm{N}
\end{array}, 1 \leq i \leq p,\right. \\
& k_{2}^{i}=\left\{\begin{array}{cc}
\frac{\eta_{i}}{h} & \frac{\eta_{i}}{h} \in \mathrm{N} \\
\left(\left[\frac{\eta_{i}}{h}\right]+1\right) & \frac{\eta_{i}}{h} \notin \mathrm{N}
\end{array}, 1 \leq i \leq m,\right.
\end{aligned}
$$

where $\left[\frac{\tau_{i}}{h}\right]$ and $\left[\frac{\eta_{i}}{h}\right]$ denote the integer part of $\frac{\tau_{i}}{h}$ and $\frac{\eta_{i}}{h}$ respectively, and $\tau=\left(\tau_{1} \tau_{2} \ldots \tau_{p}\right)^{T}$, $\eta=\left(\eta_{1} \eta_{2} \ldots \eta_{m}\right)^{T}$ are known constant vectors.

Remark 3.1 Let $\mathbf{x}(\zeta)=\sum_{j=1}^{3 k} \chi_{j}^{1}(\zeta) \mathbf{x}_{j}(\zeta)$ and $\mathbf{u}(\zeta)=\sum_{j=1}^{3 k} \chi_{j}^{2}(\zeta) \mathbf{u}_{j}(\zeta)$ where $\chi_{j}^{1}(\zeta)$ and $\chi_{j}^{2}(\zeta)$ are respectively characteristic functions of $\mathbf{x}_{j}(\zeta)$ and $\mathbf{u}_{j}(\zeta)$ for $\zeta \in\left[\zeta_{j-1}, \zeta_{j}\right]$. It is trivial that $[0,3]=\bigcup_{j=1}^{3 k} S_{j}$.

Our strategy is to using Bezier curves to approximated the solutions $\mathbf{x}_{j}(\zeta)$ and $\mathbf{u}_{j}(\zeta)$ by $\mathbf{v}_{j}(t)$ and $\mathbf{w}_{j}(\zeta)$ respectively, where $\mathbf{v}_{j}(\zeta)$ and $\mathbf{w}_{j}(\zeta)$ are given below. Individual Bezier curves which are defined over the subintervals are joined together to form the Bezier spline curves. For $j=1,2, \ldots, 3 k$, the Bezier polynomials of degree $n$ is defined that approximate the actions of $\mathbf{x}_{j}(\zeta)$ and $\mathbf{u}_{j}(\zeta)$ over the interval $\left[\zeta_{j-1}, \zeta_{j}\right]$ as follows

$$
\begin{aligned}
& \mathbf{v}_{j}(\zeta)=\sum_{r=0}^{n} \mathbf{a}_{r}^{j} B_{r, n}\left(\frac{\zeta-\zeta_{j-1}}{h}\right), \\
& \mathbf{w}_{j}(\zeta)=\sum_{r=0}^{n} \mathbf{b}_{r}^{j} B_{r, n}\left(\frac{\zeta-\zeta_{j-1}}{h}\right),
\end{aligned}
$$

where

$$
B_{r, n}\left(\frac{\xi-\xi_{j-1}}{h}\right)=\left(\begin{array}{l}
n \\
r
\end{array}\right) \frac{1}{h^{n}}\left(\xi_{j}-\xi\right)^{n-r}\left(\xi-\xi_{j-1}\right)^{r}
$$


is the Bernstein polynomial of degree $n$ over the interval $\left[\zeta_{j-1}, \zeta_{j}\right], \mathbf{a}_{r}^{j}$ and $\mathbf{b}_{r}^{j}$ are respectively $p$ and $m$ ordered vectors from the control points (see [12]). By substituting (19) in (18), $R_{1, j}(\zeta)$ for $\zeta \in\left[\zeta_{j-1}, \zeta_{j}\right]$ can be defined as

$$
\begin{aligned}
& R_{1, j}(\zeta)=\chi_{[0,1)}\left(y_{1}-t_{0}\right) L\left(\mathbf{x}_{j}(\zeta), \mathbf{u}_{j}(\zeta)\right)+\chi_{[1,2)}\left(y_{2}-y_{1}\right) L\left(\mathbf{x}_{j}(\zeta), \mathbf{u}_{j}(\zeta)\right) \\
& +\chi_{[2,3]}\left(t_{f}-y_{2}\right) L\left(\mathbf{x}_{j}(\zeta), \mathbf{u}_{j}(\zeta)\right), j=1,2, \ldots, 3 k .
\end{aligned}
$$

Let $\mathbf{v}(\zeta)=\sum_{j=1}^{3 k} \chi_{j}^{1}(\zeta) \mathbf{v}_{j}(\zeta)$ and $\mathbf{w}(\zeta)=\sum_{j=1}^{3 k} \chi_{j}^{2}(\zeta) \mathbf{w}_{j}(\zeta)$ where $\chi_{j}^{1}(\zeta)$ and $\chi_{j}^{2}(\zeta)$ are respectively characteristic functions of $\mathbf{v}_{j}(\zeta)$ and $\mathbf{w}_{j}(\zeta)$ for $\zeta \in\left[\zeta_{j-1}, \zeta_{j}\right]$. Beside the boundary conditions on $\mathbf{v}(\zeta)$, at each node, we need to impose continuity condition on each successive pair of there are also continuity constraints imposed on each successive pair of $\mathbf{v}_{j}(\zeta)$ to guarantee the smoothness. Since, the differential equation is of first order, the continuity of $\mathbf{x}$ ( or $\mathbf{v})$ and its first derivative gives

$$
\mathbf{v}_{j}^{(s)}\left(\zeta_{j}\right)=\mathbf{v}_{j+1}^{(s)}\left(\zeta_{j}\right), s=0,1, j=1,2, \ldots, 3 k-1 .
$$

Thus, the vector of control points $\mathbf{a}_{r}^{j}(r=0,1, n-1, n)$ must satisfy (see Appendix)

$$
\begin{aligned}
& \mathbf{a}_{n}^{j}\left(\zeta_{j}-\zeta_{j-1}\right)^{n}=\mathbf{a}_{0}^{j+1}\left(\zeta_{j+1}-\zeta_{j}\right)^{n}, \\
& \left(\mathbf{a}_{n}^{j}-\mathbf{a}_{n-1}^{j}\right)\left(\zeta_{j}-\zeta_{j-1}\right)^{n-1}=\left(\mathbf{a}_{1}^{j+1}-\mathbf{a}_{0}^{j+1}\right)\left(\zeta_{j+1}-\zeta_{j}\right)^{n-1} .
\end{aligned}
$$

One may recall that $\mathbf{a}_{r}^{j}$ is an $p$ ordered vector. This approach is called the subdivision scheme (or hrefinement in the finite element literature).

Remark 3.2 By considering the $C^{1}$ continuity of $\mathbf{w}$, the following constraints will be added to constraints (20),

$$
\begin{aligned}
& \mathbf{b}_{n}^{j}\left(\zeta_{j}-\zeta_{j-1}\right)^{n}=\mathbf{b}_{0}^{j+1}\left(\zeta_{j+1}-\zeta_{j}\right)^{n}, \\
& \left(\mathbf{b}_{n}^{j}-\mathbf{b}_{n-1}^{j}\right)\left(\zeta_{j}-\zeta_{j-1}\right)^{n-1}=\left(\mathbf{b}_{1}^{j+1}-\mathbf{b}_{0}^{j+1}\right)\left(\zeta_{j+1}-\zeta_{j}\right)^{n-1},
\end{aligned}
$$

where the so-called $\mathbf{b}_{r}^{j}(r=0,1, n-1, n)$ is a $m$ ordered vector.

Now, the residual function is defined in $S=\bigcup_{j=1}^{3 k} S_{j}$ as follows

$$
R=\sum_{j=1}^{3 k}\left(C_{j}\right)^{2}+\sum_{j=1}^{3 k} \int_{\zeta_{j-1}}^{\zeta_{j}}\left(R_{1, j}(\zeta)\right)^{2} d \zeta .
$$

Our aim is to solving the following problem over $S=\bigcup_{j=1}^{\beta k} S_{j}$ :

$$
\begin{aligned}
& \underset{\text { S.t. }}{\min } \quad R \\
& \frac{d \mathbf{v}_{j}}{d \zeta}=\chi_{[0,1)}\left(y_{1}-t_{0}\right) F_{1}\left(\mathbf{v}(\zeta), \mathbf{w}(\zeta), v_{1}^{-k_{1}^{1}+j}\left(\zeta-\tau_{1}\right), \ldots, v_{p}^{-k_{1}^{p}+j}\left(\zeta-\tau_{p}\right)\right. \\
& \left., w_{1}^{-k_{2}^{1}+j}\left(\zeta-\eta_{1}\right), \ldots, w_{m}^{-k_{2}^{m}+j}\left(\zeta-\eta_{m}\right)\right), j=1,2, \ldots, k, \zeta \in S_{j}, \\
& \frac{d \mathbf{v}_{j}}{d \zeta}=\chi_{[1,2)}\left(y_{2}-y_{1}\right) G_{1}\left(\mathbf{v}(\zeta), \mathbf{u}(\zeta), v_{1}^{-k_{1}^{1}+j}\left(\zeta-\tau_{1}\right), \ldots, v_{p}^{-k_{1}^{p}+j}\left(\zeta-\tau_{p}\right)\right. \\
& \left.\quad, w_{1}^{-k_{2}^{1}+j}\left(\zeta-\eta_{1}\right), \ldots, w_{m}^{-k_{2}^{m}+j}\left(\zeta-\eta_{m}\right)\right), j=k+1, \ldots, 2 k, \zeta \in S_{j},
\end{aligned}
$$




$$
\begin{aligned}
& \frac{d \mathbf{v}_{j}}{d \zeta}=\chi_{[2,3]}\left(t_{f}-y\right) Q_{1}\left(\mathbf{v}(\zeta), \mathbf{u}(\zeta), v_{1}^{-k_{1}^{1}+j}\left(\zeta-\tau_{1}\right), \ldots, v_{p}^{-k_{1}^{p}+j}\left(\zeta-\tau_{p}\right)\right. \\
& \left.\quad, w_{1}^{-k_{2}^{1}+j}\left(\zeta-\eta_{1}\right), \ldots, w_{m}^{-k_{2}^{m}+j}\left(\zeta-\eta_{m}\right)\right), j=2 k+1, \ldots, 3 k, \zeta \in S_{j}, \\
& \frac{d y_{1}}{d \zeta}=0, \zeta \in[0,2], \\
& \frac{d y_{2}}{d \zeta}=0, \zeta \in[1,3], \\
& \mathbf{a}_{n}^{j}\left(\zeta_{j}-\zeta_{j-1}\right)^{n}=\mathbf{a}_{0}^{j+1}\left(\zeta_{j+1}-\zeta_{j}\right)^{n}, j=1,2, \ldots, 3 k-1 \\
& \left(\mathbf{a}_{n}^{j}-\mathbf{a}_{n-1}^{j}\right)\left(\zeta_{j}-\zeta_{j-1}\right)^{n-1}=\left(\mathbf{a}_{1}^{j+1}-\mathbf{a}_{0}^{j+1}\right)\left(\zeta_{j+1}-\zeta_{j}\right)^{n-1}, j=1,2, \ldots, 3 k-1 \\
& \mathbf{v}_{j}\left(\zeta+t_{0}\right)=\phi\left(\zeta+t_{0}\right), \zeta \leq 0, \\
& \mathbf{w}_{j}\left(\zeta+t_{0}\right)=\psi\left(\zeta+t_{0}\right), \zeta \leq 0,
\end{aligned}
$$

The optimal control problem is converted into a nonlinear programming problem (NLP), which can be solved by known algorithms. In this paper the MATLAB optimization routine FMINCON is used for solving resulting NLP.

Ghomanjani, et al. [12] shown the convergence of the subdivision scheme as the interval width approaches zero.

Note 2: In problem (3), if $\mathbf{x}\left(t_{f}\right)$ is unknown, then we set $C_{3 k}=0$.

\section{Numerical example}

In applying the method, in Example 4.1, the Bezier curves are chosen as piecewise polynomials of degree 3 .

Example 4.1We consider a Optimal control of switched system with time delay ([27]),

$$
\begin{aligned}
& \min I=\left(x_{1}(1)-\frac{1}{2}\right)^{2}+\left(x_{2}(1)-\frac{1}{4}\right)^{2} \\
& \left\{\begin{array}{l}
\frac{d x_{1}(t)}{d t}=2 x_{1}(t) x_{2}(t)+x_{2}(t-0.1), \\
\frac{d x_{2}(t)}{d t}=3 x_{1}(t)+4 x_{2}(t-0.1), \\
\left\{\begin{array}{l}
\frac{d x_{1}(t)}{d t}=-2 x_{1}(t) x_{2}(t)+\sin \left(x_{2}(t-0.1)\right), \\
\frac{d x_{2}(t)}{d t}=3 x_{1}(t) x_{2}(t)+x_{1}(t-0.1) x_{2}(t-0.1),
\end{array}\right. \\
\left\{\begin{array}{l}
d x_{1}(t) \\
d t
\end{array}=t^{2}-2 x_{1}(t)+3 x_{2}(t-0.1),\right. \\
\frac{d x_{2}(t)}{d t}=-x_{2}(t)+x_{1}(t-0.1) x_{2}(t-0.1),
\end{array}\right.
\end{aligned}
$$




$$
x_{1}(t)=t-1, x_{2}(t)=t^{2}+1,-0.1 \leq t \leq 0
$$

Let $k=10$ and $n=3$. From (22), the following approximated solutions can be found for $x_{1}(t)$ and $x_{2}(t)$,

$$
x_{1}(t)=\left\{\begin{array}{cc}
-1974.2000 t^{3}+298.32000 t^{2}-0.09000000 t-1, & 0 \leq t \leq 0.1, \\
0.1392000(5 t-1)^{2}(10 t-1), & 0.1 \leq t \leq 0.2, \\
0, & 0.2 \leq t \leq 0.3, \\
0, & 0.3 \leq t \leq 0.4, \\
12.400000 t^{3}-16.110000 t^{2}+6.9360000 t-.99040000, & 0.4 \leq t \leq 0.5, \\
30.300000 t^{3}-49.710000 t^{2}+27.108000 t-4.9139000, & 0.5 \leq t \leq 0.6, \\
42.300000 t^{3}-82.170000 t^{2}+53.100000 t-11.415500, & 0.6 \leq t \leq 0.7, \\
56.200000 t^{3}-126.09000 t^{2}+94.158000 t-23.403000, & 0.7 \leq t \leq 0.8, \\
72 t^{3}-183.18000 t^{2}+155.16600 t-43.76140000, & 0.8 \leq t \leq 0.9, \\
541.20000 t^{3}-1469.4000 t^{2}+1330.2090 t-401.50870, & 0.9 \leq t \leq 1,
\end{array}\right.
$$

$$
x_{2}(t)=\left\{\begin{array}{cc}
1967.9000 t^{3}-297.69000 t^{2}+0.090000000 t+1, & 0 \leq t \leq 0.1, \\
-.16440000(5 t-1)^{2}(10 t-1), & 0.1 \leq t \leq 0.2, \\
0, & 0.2 \leq t \leq 0.3, \\
.20000000 t^{3}-.21000000 t^{2}+0.0720000 t-0.0081000, & 0.3 \leq t \leq 0.4, \\
.40000000 t^{3}-.54000000 t^{2}+.24000000 t-0.0353000, & 0.4 \leq t \leq 0.5, \\
.50000000 t^{3}-.84000000 t^{2}+.46800000 t-0.0868000, & 0.5 \leq t \leq 0.6, \\
.50000000 t^{3}-.99000000 t^{2}+.65100000 t-.14260000, & 0.6 \leq t \leq 0.7, \\
.20000000 t^{3}-.45000000 t^{2}+.33600000 t-0.0838000, & 0.7 \leq t \leq 0.8, \\
-.60000000 t^{3}+1.5300000 t^{2}-1.2960000 t+.364200, & 0.8 \leq t \leq 0.9, \\
249.50000 t^{3}-673.56000 t^{2}+606.12300 t-181.81290, & 0.9 \leq t \leq 1,
\end{array}\right.
$$

The graphs of approximated state $x_{1}($.$) and x_{2}($.$) are shown in Figures 1$ and 1 , respectively. The obtained results are $T_{1}=0.0886, T_{2}=0.5004$, and the optimal terminal cost is $I=10^{-7}$ when Wu, et al. [27] obtained $T_{1}=0.1500, T_{2}=0.5211$, and $I=0.0128$. 
approximate $x_{1}(t)$

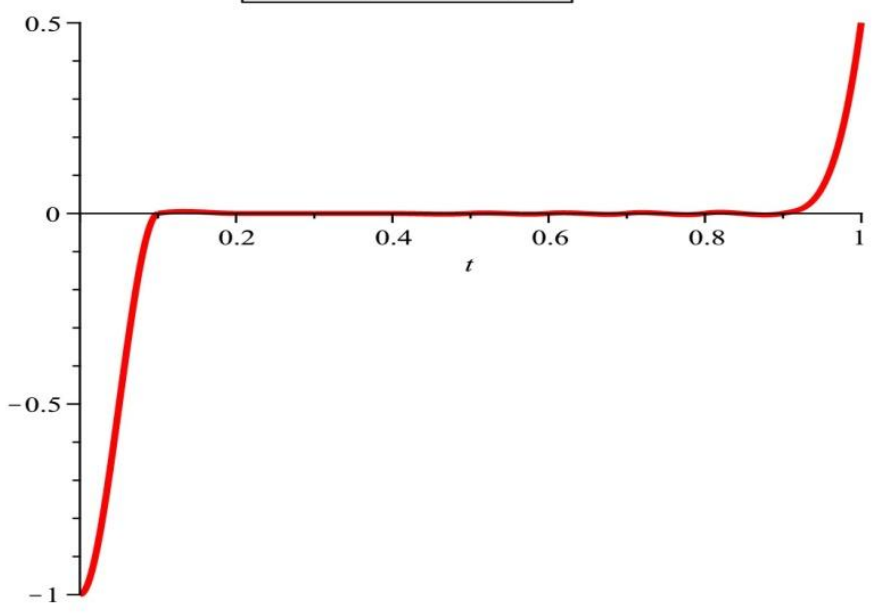

Figure 1: The graph of approximated trajectory $x_{1}(t)$

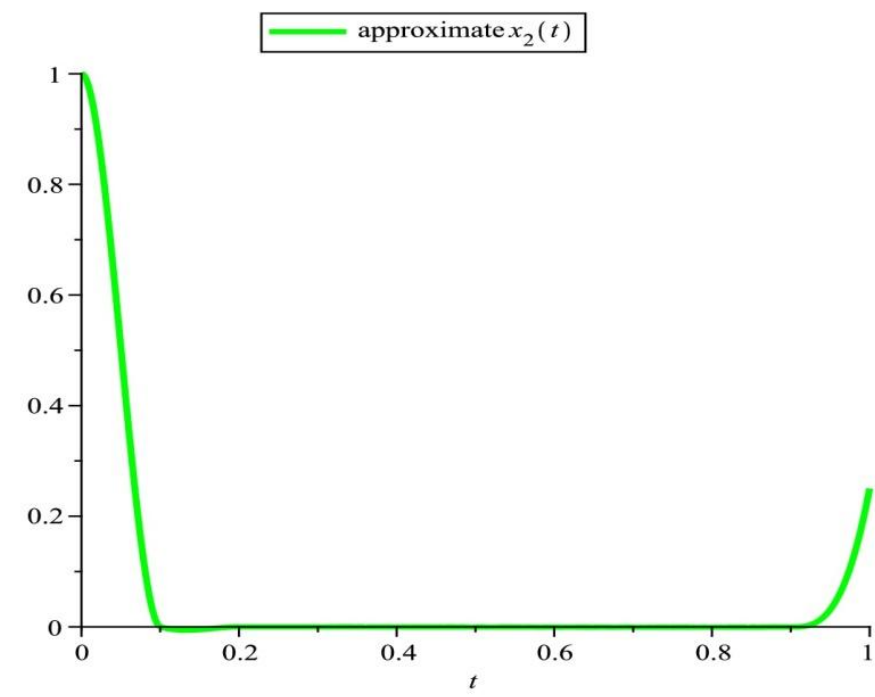

Figure 2: The graph of approximated trajectory $x_{2}(t)$

\section{Conclusions}

The optimal control problem of switched system is considered for a nonlinear system with multiple state and input delays and a quadratic criterion. The originial problem is converted into a nonlinear programming problem (NLP) by applying Bezier control points method, whereas the MATLAB optimization routine FMINCON is used for solving resulting NLP. Numerical example shows that the proposed method is efficient and very easy to use.

\section{Appendix}

\subsection{Appendix}


In this Appendix, we specify the derivative of Bezier curve.

By (19), we have

$$
v_{j}(t)=\sum_{i=0}^{n} a_{i}^{j} B_{i, n}(t), t \in[0,1]
$$

where $B_{i, n}(t)=\frac{n !}{i !(n-i) !} t^{i}(1-t)^{n-i}$. Now, we have (see [24])

$$
\frac{d B_{i, n}(t)}{d t}=n\left(B_{i-1, n-1}(t)-B_{i, n-1}(t)\right)
$$

where $B_{-1, n-1}(t)=B_{n, n-1}(t)=0$, and

$$
\begin{aligned}
& B_{i-1, n-1}(t)=\frac{(n-1) !}{(i-1) !(n-i) !} t^{i-1}(1-t)^{n-i}, \\
& B_{i, n-1}(t)=\frac{(n-1) !}{i !(n-i-1) !} t^{i}(1-t)^{n-i-1} .
\end{aligned}
$$

By using (23), the first derivative $\mathbf{v}_{j}(t)$ is shown as:

$$
\begin{aligned}
& \frac{d \mathbf{v}_{j}(t)}{d t}=\sum_{i=1}^{n-1} n \mathbf{a}_{i}^{j} B_{i-1, n-1}(t)-\sum_{i=0}^{n-1} n \mathbf{a}_{i}^{j} B_{i, n-1}(t) \\
& =\sum_{i=0}^{n-1} n \mathbf{a}_{i+1}^{j} B_{i, n-1}(t)-\sum_{i=0}^{n-1} n \mathbf{a}_{i}^{j} B_{i, n-1}(t) \\
& =\sum_{i=0}^{n-1} B_{i, n-1}(t) n\left\{\mathbf{a}_{i+1}^{j}-\mathbf{a}_{i}^{j}\right\}
\end{aligned}
$$

\subsection{Appendix}

Now, we specify the procedure of derivation (21) from (20).

By (19), we have

$$
\begin{aligned}
& \mathbf{v}_{j}(t)=\left(\begin{array}{l}
n \\
0
\end{array}\right) \mathbf{a}_{0}^{j} \frac{1}{h^{n}}\left(t_{j}-t\right)^{n}+\ldots+\left(\begin{array}{l}
n \\
n
\end{array}\right) \mathbf{a}_{n}^{j} \frac{1}{h^{n}}\left(t-t_{j-1}\right)^{n}, \\
& \mathbf{v}_{j+1}(t)=\left(\begin{array}{l}
n \\
0
\end{array}\right) \mathbf{a}_{0}^{j+1} \frac{1}{h^{n}}\left(t_{j+1}-t\right)^{n}+\ldots+\left(\begin{array}{l}
n \\
n
\end{array}\right) \mathbf{a}_{n}^{j+1} \frac{1}{h^{n}}\left(t-t_{j}\right)^{n},
\end{aligned}
$$

by substituting $t=t_{j}$ into (25) and (26), one have

$$
\begin{aligned}
& \mathbf{v}_{j}\left(t_{j}\right)=\mathbf{a}_{n}^{j} \frac{1}{h^{n}}\left(t_{j}-t_{j-1}\right)^{n}, \\
& \mathbf{v}_{j+1}\left(t_{j}\right)=\mathbf{a}_{0}^{j+1} \frac{1}{h^{n}}\left(t_{j+1}-t_{j}\right)^{n} .
\end{aligned}
$$

To preserve the continuity of Bezier curves at the nodes, one needs to impose the condition $\mathbf{v}_{j}\left(t_{j}\right)=\mathbf{v}_{j+1}\left(t_{j}\right)$, so from (27) and (28), we have

$$
\mathbf{a}_{n}^{j}\left(t_{j}-t_{j-1}\right)^{n}=\mathbf{a}_{0}^{j+1}\left(t_{j+1}-t_{j}\right)^{n} .
$$

From (24), the first derivatives of $\mathbf{v}_{j}(t)$ and $\mathbf{v}_{j+1}(t)$ are respectively as: 


$$
\begin{aligned}
& \frac{d \mathbf{v}_{j}(t)}{d t}=\sum_{i=0}^{n-1} B_{i, n-1}(t) n\left(\mathbf{a}_{i+1}^{j}-\mathbf{a}_{i}^{j}\right) \\
& =\sum_{i=0}^{n-1}\left(\begin{array}{l}
n-1 \\
i
\end{array}\right)\left(t_{j}-t\right)^{n-1-i}\left(t-t_{j-1}\right)^{i} \frac{1}{h^{n}}\left\{n\left(\mathbf{a}_{i+1}^{j}-\mathbf{a}_{i}^{j}\right)\right\} \\
& =\left(\begin{array}{l}
n-1 \\
0
\end{array}\right)\left\{n\left(\mathbf{a}_{1}^{j}-\mathbf{a}_{0}^{j}\right)\right\} \frac{1}{h^{n}}\left(t_{j}-t\right)^{n-1} \\
& +\ldots+\left(\begin{array}{l}
n-1 \\
n-1
\end{array}\right)\left\{n\left(\mathbf{a}_{n}^{j}-\mathbf{a}_{n-1}^{j}\right)\right\} \frac{1}{h^{n}}\left(t-t_{j-1}\right)^{n-1}, \\
& \frac{d \mathbf{v}_{j+1}(t)}{d t}=\sum_{i=0}^{n-1}\left(\begin{array}{l}
n-1 \\
i
\end{array}\right)\left(t_{j+1}-t\right)^{n-1-i}\left(t-t_{j}\right)^{i} \frac{1}{h^{n}}\left\{n\left(\mathbf{a}_{i+1}^{j+1}-\mathbf{a}_{i}^{j+1}\right)\right\} \\
& =\left(\begin{array}{l}
n-1 \\
0
\end{array}\right)\left\{n\left(\mathbf{a}_{1}^{j+1}-\mathbf{a}_{0}^{j+1}\right)\right\} \frac{1}{h^{n}}\left(t_{j+1}-t\right)^{n-1} \\
& +\ldots+\left(\begin{array}{l}
n-1 \\
n-1
\end{array}\right)\left\{n\left(\mathbf{a}_{n}^{j+1}-\mathbf{a}_{n-1}^{j+1}\right)\right\} \frac{1}{h^{n}}\left(t-t_{j}\right)^{n-1} .
\end{aligned}
$$

By substituting $t=t_{j}$ into (30) and (31), we have

$$
\begin{aligned}
& \frac{d \mathbf{v}_{j}\left(t_{j}\right)}{d t}=n\left(\mathbf{a}_{n}^{j}-\mathbf{a}_{n-1}^{j}\right) \frac{1}{h^{n}}\left(t_{j}-t_{j-1}\right)^{n-1}, \\
& \frac{d \mathbf{v}_{j+1}\left(t_{j}\right)}{d t}=n\left(\mathbf{a}_{1}^{j+1}-\mathbf{a}_{0}^{j+1}\right) \frac{1}{h^{n}}\left(t_{j+1} t_{j}\right)^{n-1},
\end{aligned}
$$

and to preserve the continuity of the first derivative of Bezier curves at nodes, by equalizing (32) and (33), we have

$$
\left(\mathbf{a}_{n}^{j}-\mathbf{a}_{n-1}^{j}\right)\left(t_{j}-t_{j-1}\right)^{n-1}=\left(\mathbf{a}_{1}^{j+1}-\mathbf{a}_{0}^{j+1}\right)\left(t_{j+1}-t_{j}\right)^{n-1}
$$

\section{References}

[1] J. V. Beltran and J. Monterde, "Bezier solutions of the wave equation," Lecture notes in Computational Sciences, Computational Science and its Applications-ICCSA (A. Laganà, M. L. Gavrilova, V. Kumar, Y. Mun, C. J. K.Tan \& O. Gervasi eds). Lecture Notes in Computer Science, vol. 3044. Berlin: Springer, pp. 631-640, 2004.

[2] A. Bemporad, A. Giua, and C. Seatzu, "Synthesis of state-feedback optimal controllers for continuous-time switched linear systems," In Proc. 41st IEEE conference on decision and control, Las Vegas, Nevada USA, 2002.

[3] R. Cholewa, A. J. Nowak, R. A. Bialecki, and L. C. Wrobel, "Cubic Bezier splines for BEM heat transfer analysis of the 2-D continuous casting problems," Computational Mechanics, vol. 28, pp. 282-290, 2002.

[4] C. H. Chu, C. C. L. Wang, and C. R. Tsai, "Computer aided geometric design of strip using developable Bezier patches," Computers in Industry, vol. 59, no. 6, pp. 601-611, 2008.

[5] M. Egerstedt, Y. Wardi, and F. Delmotte, "Optimal control of switching times in switched dynamical systems," In Proc. IEEE conference on decision and control, vol. 3, pp. 2138-2143, 2003. 
[6] M. Evrenosoglu and S. Somali, "Least squares methods for solving singularity perturbed two-points boundary value problems using Bezier control point," Applied Mathematics Letters, vol. 21, no. 10, pp. 1029-1032, 2008.

[7] G. E. Farin, "Curve and surfaces for computer aided geometric design," First ed, New York: Academic Press, 1988.

[8] B. Farhadinia, K. L. Teo, and R. C. Loxton, "A computational method for a class of non-standard time optimal control problems involving multiple time horizons," Mathematical Computer Modelling, vol. 49, pp. 1682-1691, 2009.

[9] M. Gachpazan, "Solving of time varying quadratic optimal control problems by using Bezier control points," Computational and Applied Mathematics, vol. 30, no. 2, pp. 367-379, 2011.

[10] J. Geromela, P. Colaneri, and P, Bolzern, "Passivity of switched linear systems: Analysis and control design," Systems and Control Letters, vol. 61, pp. 549-554, 2012.

[11] F. Ghomanjani, M. H. Farahi, “ The Bezier control points method for solving delay differential equation," Intelligent Control and Automation, vol. 3, no. 2, pp. 188-196, 2012.

[12] F. Ghomanjani, M. H. Farahi and M. Gachpazan, "Bezier control points method to solve constrained quadratic optimal control of time varying linear systems," Computational and Applied Mathematics, vol. 31, no. 3, pp. 1-24, 2012.

[13] A. Giua, C. Seatzu, and C. Van Der Me, "Optimal conrol of switched autonomous linear systems," In Proc. IEEE conference on decision and control, pp. 2472-2477, 2001.

[14] K. Harada and E. Nakamae, "Application of the Bezier curve to data interpolation, Computer-Aided Design," International Journal of Computer Mathematics, vol. 14, no. 1, pp. 55-59, 1982.

[15] M. Heinkenschloss, "A time-domain decomposition iterative method for the solution of distributed linear quadratic optimal control problems," Applied Mathematics and Computation, vol. 173, pp. 169-198, 2005.

[16] I. Hwang, J. Li, and D. Du, "A numerical algorithm for optimal control of a class of hybrid systems: differential transformation based approach,” International Journal of Control, vol. 81, no. 2, pp. 277-293, 2008.

[17] H. Juddu, "Spectral method for constrained linear-quadratic optimal control," Mathematics Computers In simulation, vol. 58, pp. 159-169, 2002.

[18] B. Lang, "The synthesis of wave forms using Bezier curves with control point modulation," In: The Second CEMS Research Student Conference, 1st edn. Morgan kaufamann, San Francisco, 2004.

[19] A. T. Layton and M. Van de Panne, "A numerically evident and stable algorithm for animating water waves," The visual Computer, vol. 18, pp. 41-53, 2002.

[20] M. Kamgarpour, and C. Tomlin, "On optimal control of non-autonomous switched systems with a fixed mode sequence," Automatica, Doi:10.1016/j.automatica, 2012.03.019, 2012.

[21] M. Margaliot, "Stability analysis of switched systems using variational principles: An introduction," Automatica, vol. 42, pp. 2059-2077, 2006.

[22] B. Niua, and J. Zhaoa, "Stabilization and $L_{2}$-gain analysis for a class of cascade switched nonlinear systems: An average dwell-time method," Nonlinear Analysis: Hybrid Systems, vol. 5, pp. 671-680, 2001.

[23] G. N $\ddot{u}$ rnberger and F. Zeilfelder, "Developments in bivariate spline interpolation, Journal of Computational and Applied Mathematics," Computers and Mathematics with Applications, vol. 121, no. 1-2, pp. 125-152, 2000.

[24] H. Prautzsch, W. Boehm, M. Paluszny, Bezier and B-Spline Techniques,” Springer. 2001.

[25] Y. Q. Shi, H. Sun, “Image and video compression for multimedia engineering," CRC Press LLc, 2000.

[26] R. Winkel, "Generalized Bernstein Polynomials and Bezier Curves: An Application of Umbral Calculus to Computer Aided Geometric Design,” Advances in Applied Mathematics, vol. 27, no. 1, pp. 51-81, 2001.

[27] C. Wu, K. L. Teo, R. Li, and Y. Zhao, “Optimal control of switched systems with time delay,” Appl, Math. Letters, vol. 19, pp. 1062-1067, 2006.

[28] X. Xu, G. Zhai, and S. He, "Some results on practical stabilizability of discrete-time switched affine systems," Nonlinear Analysis: Hybrid Systems, vol. 4, pp. 113-121, 2010.

[29] X. Xu, and P. J. Antsakalis, "Optimal control of switched systems based on parameterization of the switching instants," IEEE Transactions on Automatic Control, vol. 49, no. 1, pp. 2-16, 2004.

[30] J. Zheng, T. W. Sederberg, and R. W. Johnson, "Least squares methods for solving differential equations using Bezier control points,", Applied Numerical Mathematics, vol. 48, pp. 237-252, 2004. 